INVITLD PAPER

\title{
APPLICATION OF CATHODOLUMINESCENCE MICROSCOPY TO THE STUDY OF NATIVE ACCEPTORS IN GALLIUM ANTIMONIDE
}

\author{
J. Piqueras", B. Mendez ${ }^{a}$, G.N. Panin ${ }^{\mathrm{x},}$, P.S. Dutta ${ }^{\mathrm{b},+}$ \\ and E. Dieguez ${ }^{b}$ \\ 'Departamento de Fisica de Materiales, Facultad de Fisica \\ Universidad Complutense, 28040, Madrid, Spain. \\ "Institute of Microelectronics Technology and High Purity \\ Materials, Russian Academy of Sciences, Chernogolovka, \\ Moscow district 142432, Russia. \\ 'Instituto Nicolas Cabrera and Departamento de Fisica \\ de Materiales, C-IV, Universidad Autonoma, 28049, Madrid, \\ Spain. \\ ${ }^{+}$Department of Physics, Indian Institute of Science, \\ Bagalore-560 012, India.
}

\begin{abstract}
Summary
Cathodoluminescence in the scanning electron microscope is used to ivestigate growth and prosess induced defects in GaSb crystals. In particular, luminescence emission has been used to study the nature of acceptor defects present after different annealing and irradiation treatments.
\end{abstract}

\section{Introduction}

In recent years, Gallium Antimonide has generated increasing interest as a potencial substrate material for various lattice-matched devices in the bandgap range $0.3 \mathrm{eV}$ to $1.58 \mathrm{eV}$ (1). However, significant progress has to be made both in materials and processing aspects before it can be employed for device applications. It is well known that the quality of epitaxial layers and the yield of devices made from them depend on the structural perfection of the substrate. Hence, characterization of defects is of great importance for reliable device perfomance. The interest of defect studies in GaSb refers notonly to the as-grown substrate material but to the effect of different processes as doping,ion implantation, etc. For 
defect characterization, the cathodoluminescence (CL) technique has been found to be highly sensitive and is extensively used for the spatial defect mapping of various elemental and compound semiconductors. However, CL microscopy has been seldom applied to defects study in GaSb (2) (3) (4). In this paper, we present the results of $\mathrm{CL}$ investigation of defects in bulk $\mathrm{GaSb}$ generated during growth, post growth annealing treatments, doping and ion beam irradiation.

Undoped $\mathrm{GaSb}$ is always p-type in nature with the acceptor concentration of approximately $10^{17} \mathrm{~cm}^{-3}$ at room temperature. The acceptor is intrinsic and is due to vacant gallium site $\left(\mathrm{V}_{\mathrm{Ga}}\right)$ and gallium antisite $\left(\mathrm{Ga}_{\mathrm{sb}}\right)$. The acceptor concentration can be reduced either by non-stoichiometric melt growth or by employing low temperature growth techniques. In this work we have perfomed post growth annealing in vacuum, gallium and antimony atmospheres to examine the evolution and nature of the native acceptors. Due to the presence of high concentration of native acceptors, impurity doping to obtain n$\mathrm{GaSb}$ always leads to compensation (5) (6). As a result, the mobility of electrons reduces and controlled doping to very low levels poses problems. Dopant incorporation by diffusion has been given limited attention till now (1). In this paper we present results concerning the nature of luminescent centers in Tediffused GaSb. Such a study of diffusion doping is of interest for basic $p-n$ junction device structures. From the device fabrication point of view, the ion milling technique is of utmost importance and has been widely used in other materials. More limited attention has been devoted to the problems of ion implantation and related studies in GaSb (7) (8) (9) (10). In the last part of this work we have carried out ion milling of p-GaSb by $\mathrm{Ar}^{+}$ions and studied its effect on the electronic properties by $\mathrm{CL}$. Electron beam induced current (EBIC) measurements in the scanning electron microscope have been also made to complement the $\mathrm{CL}$ measurements after ion milling.

\section{Experimental Method}

The samples used in this study were vertical Bridgman grown GaSb single crystals (11). For the annealing treatments the wafers were prepared by conventional chemo-mechanical polishing. Prior to annealing, the samples were etched in $\mathrm{CH}_{3} \mathrm{COOH}: \mathrm{HNO}_{3}: \mathrm{HF}$ (20:9:1) to remove any damaged layer left behind after polishing, dipped in $\mathrm{HCl}$ and rinsed in methanol. Thermal annealing of the samples was carried out by placing the wafers in quartz ampoule under vacuum. For the gallium annealing, $6 \mathrm{~N}$ pure gallium was spread on the wafer surface at room temperature. After annealing, the gallium sticking to the surface was removed by rinsing in $\mathrm{HCl}$. For annealing in antimony atmosphere, $6 \mathrm{~N}$ pure antimony balls were kept along with the wafer. Other wafers were annealed under vacuum of $10^{-6}$ torr. The annealing temperature and time was kept constant at $500^{\circ} \mathrm{C}$ and 12 hours respectively for all the cases. The Te-diffusion experiments were carried out in evacuated and sealed quartz ampoules at $500^{\circ} \mathrm{C}$ for 2,15 
and 24 hours. Prior to diffusion, the quartz ampoules were prebacked at $800^{\circ} \mathrm{C}$ under $10^{-6}$ torr to remove any volatile contaminants. The volume of sealed ampoules was about $6 \mathrm{~cm}^{3}$. The diffusion source mass amount was approximately $100 \mathrm{mg}$. During the diffusion experiments, the wafers of $\mathrm{GaSb}$ were placed along with Te. Pieces of $\mathrm{Sb}$ were also kept to create an overpressure of $\mathrm{Sb}$ during the diffusion cycle. Diffusion was simultaneously carried out on several samples under identical conditions. At the end of diffusion, the ampoule was withdrawn from the furnace and cooled rapidly in liquid nitrogen to condense the Te vapour on the ampoule wall and thus prevent any metallic condensation on the sample surface. The wafers were rinsed in organic solvents and $\mathrm{HCl}$ before any measurements. No visible degradation of the sample surfaces occurred even after 24 hours of heat treatment. The depth profiles of the net donor concentration of varius samples were evaluated from the Capacitance-Voltage (C-V) measurements at room temperature.

The ion beam milling treatnent was perfomed in an ion beam milling unit with $4 \mathrm{kV} \mathrm{Ar}$ ions for $30 \mathrm{~min}$ at room temperature. The samples,rotating during the ion milling process, were bombarded at an angle of $45^{\circ}$ to the surface. The ion beam current density was $70 \mu \mathrm{A} / \mathrm{cm}^{2}$.

CL measurements were carried out using a Hitachi S-2500 scannig electron microscope in the emissive and $\mathrm{CL}$ modes at 77K. A liquid nitrogen cooled North Coast EO-817 germanium detector was used for the signal detection. The details of the experimental set-up for spectral and panchromatic CL measurements are presented elsewhere (12). The EBIC measurements in the electron microscope were carried out at liquid nitrogen temperature with electron beam current and voltage of $10^{-9} \mathrm{~A}$ an $25 \mathrm{kV}$ respectively. Electrical contacts for the EBIC measurements were provided by silver paste with gold wires to the treated (ion milled) region and back side of the sample.

\section{RESULTS and Discusion}

\subsection{Annealed Samples}

Fig. 1 shows the $\mathrm{CL}$ spectrum at $77 \mathrm{~K}$ of an as-grown sample. The main emission bands appear at $796 \mathrm{meV}$ and $775 \mathrm{meV}$. Weak transitions at 756 and $830 \mathrm{meV}$ can also be seen. The $796 \mathrm{meV}$ corresponds to the band-band transition and the $775 \mathrm{meV}$ (commonly known as band $\mathrm{A}$ ) to a transition from the conduction band to the neutral state of the native acceptor level $\mathrm{V}_{\mathrm{Ga}} \mathrm{Ga}^{\mathrm{sb}}(13)(14)(15)$. The peak at $756 \mathrm{meV}$ (designated as band B) has been previously attributed to a transition from the conduction band to to a triple native acceptor $\mathrm{V}_{\mathrm{Ga}} \mathrm{Ga}_{\mathrm{Sb}} \mathrm{V}_{\mathrm{Ga}}$, arising from excess of Ga-vacancies (16). However, the origin of this transition has not been clearly resolved up till now. The annealed samples exhibit only the 775 and 756 meV transitions, but with difference in relative intensities depending on the annealing conditions 
(Fig.2a). In general, the relative intensity of the $775 \mathrm{meV}$ with respect to the $756 \mathrm{meV}$

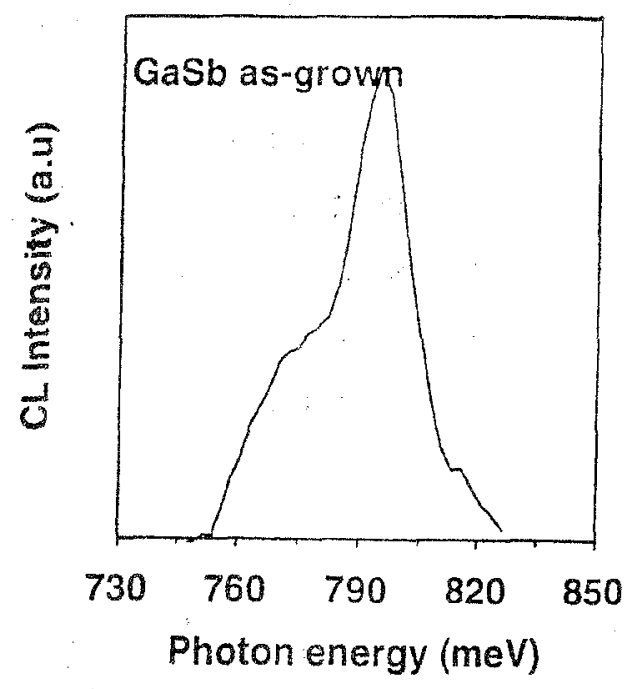

Fig. 1. Cl spectrum of as-grown GaSb
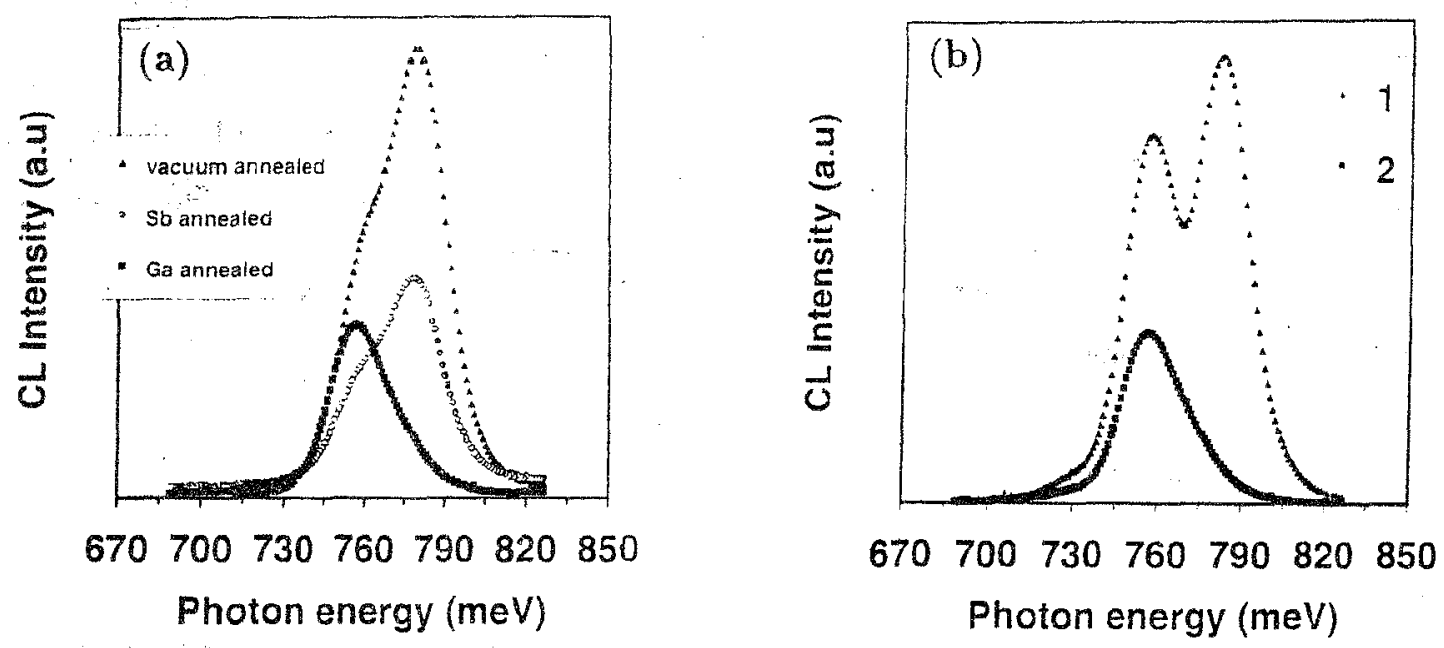

Fig. 2. Spectra of GaSb: a) after annealing in different atmospheres for 12 hours at $500 \mathrm{oC}, \mathrm{b}$ ) in different regions of a Gamannealed sample.

transition decreases after annealing. The vacuum and Sb- annealed samples show similar $\mathrm{CL}$ spectrum. Moreover, the relative intensity of the $775 \mathrm{meV}$ with respect to the $756 \mathrm{meV}$ transition is less in the Sbrich samples. In the Ga-annealed sample, the $756 \mathrm{meV}$ transition enhances drastically andat certain 
locations of the sample, it is even more intense than the $775 \mathrm{meV}$ peak as

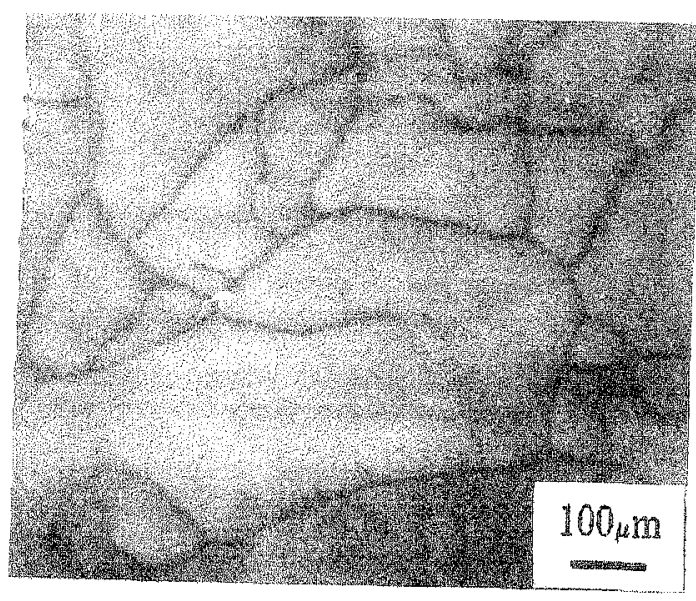

Fig. 3. $\mathrm{CL}$ image of as-grown $\mathrm{GaSb}$

shown in Fig. 2b. Thus the band B transition is probably associated with a defect involving excess gallium and can be due to a gallium antisite or a related complex. Hence the decrease in relative intensity of the $775 \mathrm{meV}$ peak compared to the $756 \mathrm{meV}$ in the $\mathrm{Sb}$ and vacuum annealed samples can be explained by considering, the reduction of the native acceptors on annealing, which shifts the Fermi level position towards the conduction band and hence the intensity of the $756 \mathrm{meV}$ transition increases. The CL image of as-growa GaSb shows dark features related to the presence of sub-boundaries in the material (Fig.3). The CL images of the Sb-annealed sample revealed dark precipitate like defects which contribute to the reduction of luminescence emission (Fig.4a). These defects are not seen in as-grown or vacuum annealed samples. The defect structure in the vacuum annealed sample is similar to that of the untreated ones. The Ga-anriealed sample exhibits a uniform CL image (see Fig.4b) without any contrast inhomogeneities(sub-boundaries etc.) as seen in the other samples. This observation suggests that postgrowth annealing in Ga-bath can be technologically important for preparing GaSb wafers with uniform properties for opto-electronic applications. On the other hand, although annealing in $\mathrm{Sb}$ atmosphere reduces the native defect concentration, precipitate formation takes place modifying the potential applications of the GaSb crystals as substrates. 

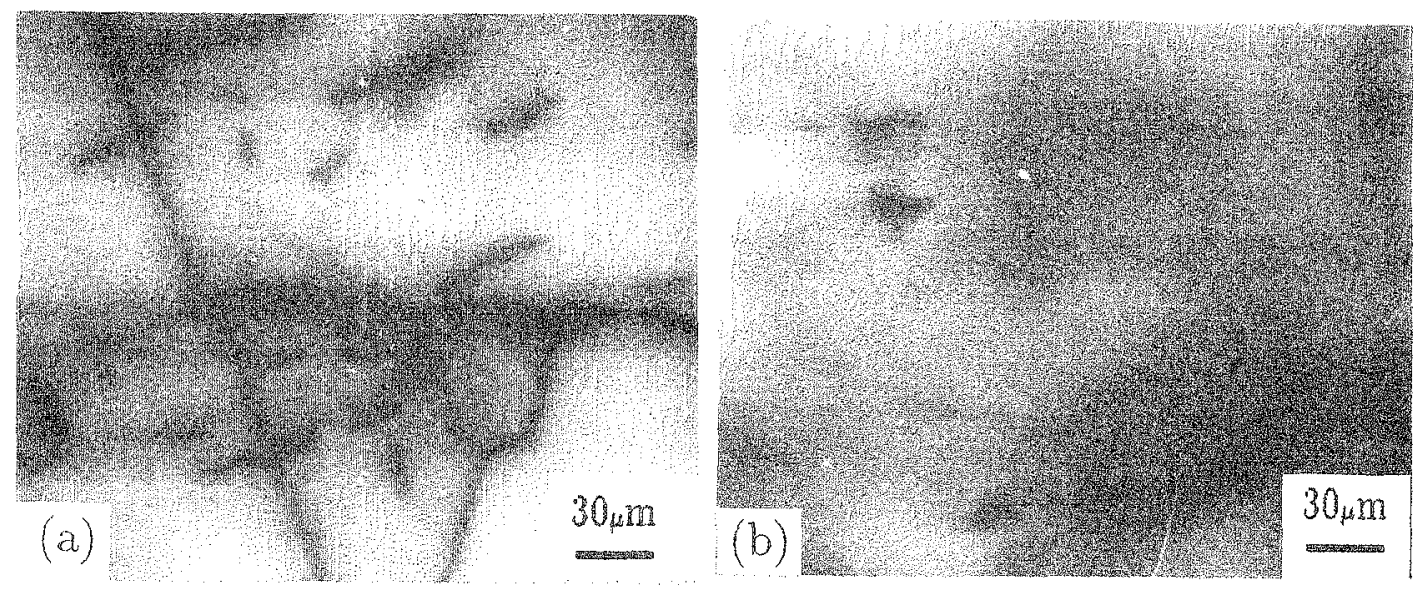

Fig. 4. CL images of $\mathrm{GaSb}$ annealed in $\mathrm{Sb}(\mathrm{a})$ and in $\mathrm{Ga}(\mathrm{b})$.

\subsection{Te-diffused samples}

CL images of Te-diffused samples are shown in Fig.5. The sample for which the diffusion was carried out for 2 hours appeared to be almost precipitate free (see Fig.5a), but small dark dots of 1-2 um size are clearly revealed. This contrast could be due to the decoration of dislocations associated with the presence of tellurium as occurs in other III-V compounds. As described above in undoped $\mathrm{GaSb}, \mathrm{Cl}$ images show dark contrast related to the presence of subboundaries in the material and the inner portion of the grains appears uniformly bright. With increase in diffusion time to 15 hours, precipitates start appearing throughout the sample. The size and concentration of the precipitates increase with further increase in diffusion time. For the sample with 24 hours of diffusion, large clusters and precipitates could be seen (Fig.5b). Characterization of extended defects in heavily Te- doped $\mathrm{CZ}$ grown $\mathrm{GaSb}$ has been previously carried out by transmission electron microscopy (17) and planar defects have been identified as $\mathrm{Ga}_{2} \mathrm{Te}_{3}$ precipitates. In our case, the precipitates can be either $\mathrm{Te}$ or $\mathrm{Ga}_{2} \mathrm{Te}_{3}$. In order to get a more accurate information CL spectra obtained from different Te-diffused samples were analyzed. The sample with shortest diffusion time exhibits luminescence peaks at 744,775 and $796 \mathrm{meV}$. The $744 \mathrm{meV}$ peak is 
intrinsic to $\mathrm{Te}$ and has been attributed to transition from conduction band to an
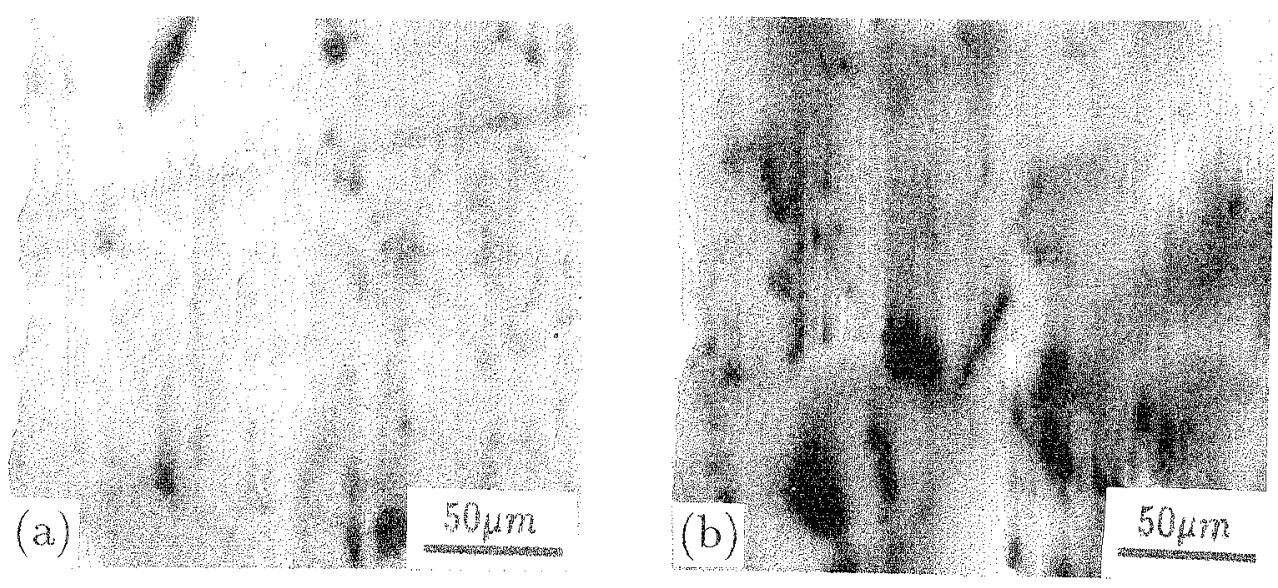

Fig. 5. CL images of Te-diffused sample after diffusion for 2 hours (a) and 24 hours (b).

acceptor state $\mathrm{V}_{\mathrm{Ga}} \mathrm{Ga}_{\mathrm{Sb}} \mathrm{Te}_{\mathrm{Sb}}$. The spatial distribution of this complex seems to be connected to dislocations as is shown in Fig. $5 \mathrm{a}$. With increase in Te concentration, the intensity of $744 \mathrm{meV}$ peak increases (Fig.6), indicating the enbancement of
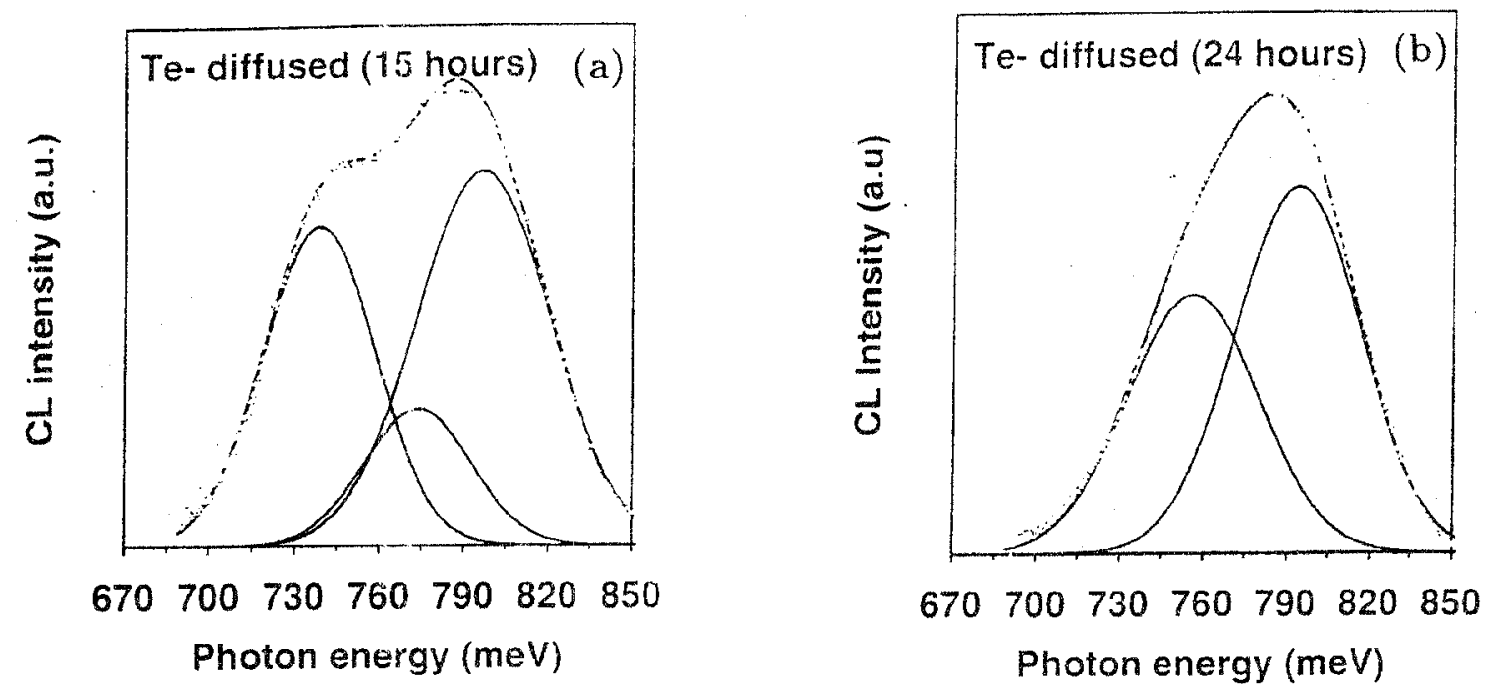

Fig. 6. CL spectra of Te-diffused samples after diffusion for 15 hours (a) and 24 hours (b). 
the formation of the related acceptor centre. In the CL spectrum of the sample with highest $\mathrm{Te}$ concentration it is observed that the dominant transition occurs at $756 \mathrm{meV}$ while the $744 \mathrm{meV}$ emission is almost disappeared. The fact that the intensity of the $756 \mathrm{meV}$ peak increases after long diffusion times can be explained by an increase in volatilization of $\mathrm{Sb}$. This in turns results in excess of $\mathrm{Ga}$ and is similar to the situation of annealing under $\mathrm{Ga}$ - rich conditions. The probability of formation of $\mathrm{Ga}_{2} \mathrm{Te}_{3}$ precipitates and the increase in concentration and size of precipitates with increasing diffusion time can also be explained by the presence of excess Ga. It appears from the above results that the dominant compensating acceptor varies with increase in diffusion time.

\subsection{Ion irradiated samples}

$\mathrm{CL}$ images show that ion milling causes an enhancement of the panchromatic $\mathrm{CL}$ emission in the irradiated area and its surroundings as shown in Fig.7a. CL intensity is higher at the center of the treated region and decreases radially. A part from the increased luminescence intensity in the irradiated regions, uniformly distributed precipitates could also be seen under higher magnification as depicted in Fig.7b. At exactly the location of a dark precipitate like in the CL image the secondary electron image exhibits precipitates like topographic features. These extended defects were absent on the
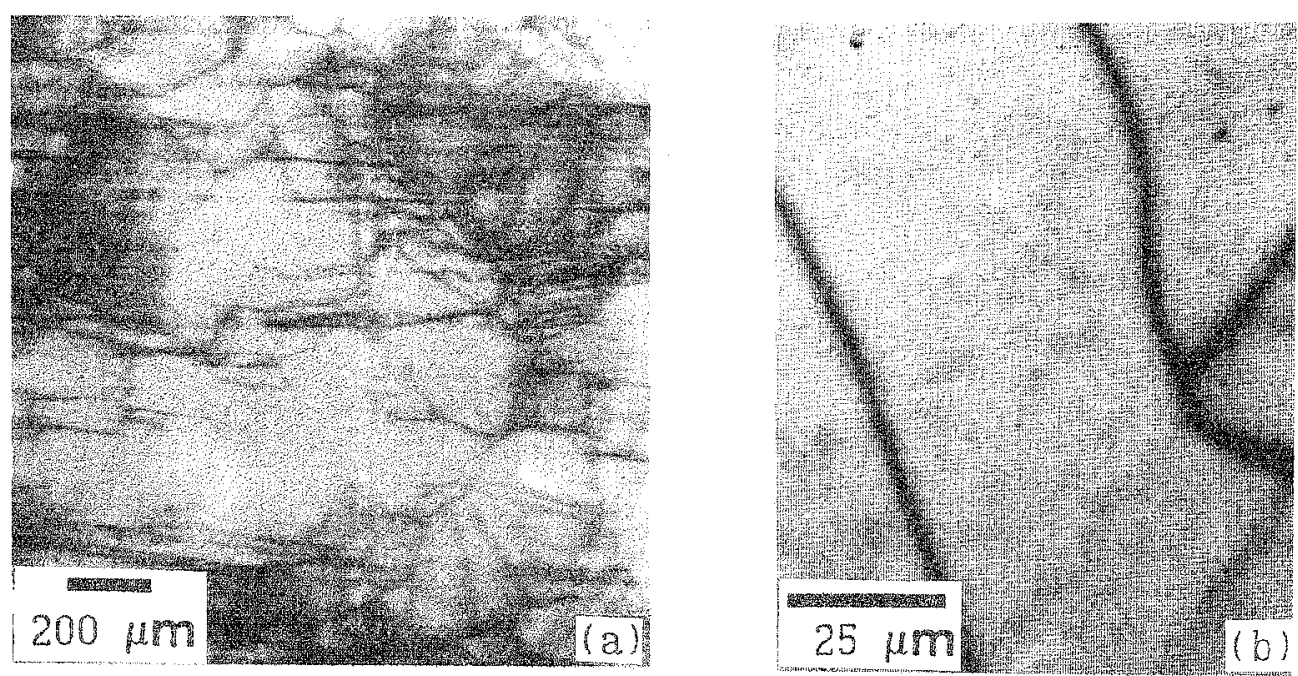

Fig. 7. CL images at the centre of the irradiated region at two magnifications.

surface of the as-grown samples and are created during the ion milling treatment. Apart from the increased luminescence no differences in the relative band intensities or spectral features of the treated 
and untreated samples could be observed. Spectra show the bands at 775 and $756 \mathrm{meV}$ associated with the native acceptors $\mathrm{V}_{\mathrm{Ga}}$ and $\mathrm{Sa}_{\mathrm{Sb}}$. The increase in $\mathrm{CL}$ intensity after ion milling can be explained either by a bombardment induced increase in acceptor concentration or by the reduction of non-radiative recombination centers at grain boundaries or dislocations. In order to study these possibilities and in general to investigate the effect of ion milling on the electronic properties of $\mathrm{GaSb}$, the spatial resolved EBIC technique in the scauning elctron microscope was used. EBIC measurements with metal contact on $\mathrm{p}$ - GaSb cannot be carried out due to the low barrier height produced and therefore to the low efficiency of excess charge carriers collection. However, if as in other semiconductors, ion milling causes local p to n-type conversion EBIC measurements would be possible. Fig. 8 shows a typical EBIC image of the ion irradiated
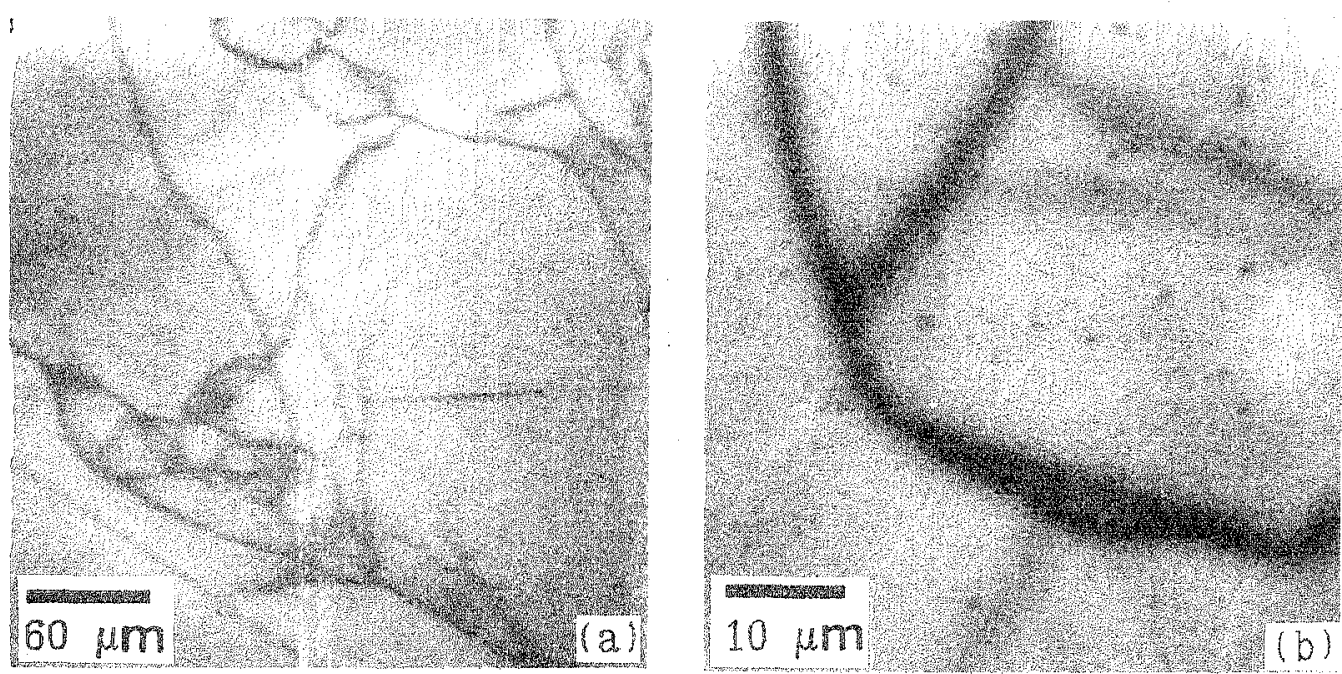

Fig. 8. EBIC images of GaSb after irradiation at two magnifications.

sample. The detection of ERIC contrast implies a type conversion caused by the ion milling treatment. The untreated samples did not show any EBIC signal.The polarity of the EBIC signal corresponds to the formation of a n-type surface layer. This result enables to rule out the possibility that the CL spectral changes after ion milling are due to the increase of acceptor concentration because such increase would not lead to an $\mathbf{n}$-type surface region. The reduction of non-radiative recombination centers during ion milling seems to be a probable cause of the observed $\mathrm{CL}$ intensity increase. 


\section{Conclusions}

CL mode in the scanning electron microscope is a suitable technique that enables to study the morphology and nature of growth and process induced defects in GaSb with high spatial resolution.CL images reveal that defect distribution in as-grown crystals is inhomogenous and annealing in vacuum , $\mathrm{Sb}$ and $\mathrm{Ga}$ atmospheres causes an increase in homogeneity. Ga-annealed samples exhibit featureless $\mathrm{CL}$ images suggesting an uniform nature of the luminescent defect distribution. In these samples it has been found that a luminescence band at $756 \mathrm{meV}$, with an unclear origin till now, is related to excess $\mathrm{Ga}$ in the sample. Te diffusion provides new information about defects in GaSb. During Te-difussion the type of defects formed in the samples is a function of diffusion time. CL images show that this is a consequence of precipitation processes. Ion beam milling causes also an increase of the luminescence in $\mathrm{GaSb}$ as well as the type conversion from $\mathrm{p}$ to $\mathrm{n}$ of the subsurface layer as revealed by EBIC measurements.

\section{References}

1) A.G. Milnes and A.Y. Polyakov, Solid State Electronics 36, 803 (1993)

2) B. Méndez, P.S. Dutta, J. Piqueras and E. Dieguez, Appl. Phys. Lett. 67, 2648 (1995)

3) G.N. Panin, P.S. Dutta, J. Piqueras and E. Dieguez, Appl. Phys. Lett. 67, 3584 (1995)

4) P.S. Dutta, A.K. Sreedhar, H.L. Bhat, G.C. Duber, V. Kumar, E. Dieguez, U. Pal and J. Piqueras, Accepted in Journal of Applied Physics

5) K. Nakashima, Jpn. J. Appl. Phys. 20, 1085 (1981)

6) A. I. Lebedev and I.A. Strel'nikova, Sov. Phys. Semicond. 13, 229 (1979)

7) R.Callec, P.N. Favennec, M.Salvi, H.L. Haridon, and M.Gauneau, Appl.Phys.Lett.59, 1872 (1991)

8) S.J. Pearton, A.R. Von Neida, J. M. Brown, K.T. Short, L. J. Oster, and U.K. Chakrabarti, J. Appl. Phys. 64, 629 (1988)

9) M. Perotin, L. Gouskov, H. Luquet, P. Abiale Abi, A. Sabir, and A. Pérez, J. Appl. Phys. $6 \mathbf{8}, 3756$ (1990)

10) Y. K. Su, K.J. Gan, J.S. Hwang, and S.L. Tyan, J. Appl. Phys. 68, 5584 (1990)

11) P.S. Dutta, K.S. Sangunni, H.L. Bhat and V. Kumar, J. Cryst. Growth. 141, 44 (1994)

12) B. Méndez and J. Piqueras, J. Appl. Phys. 69, 2776 (1991)

13) W. Jakowetz, W. Ruhle, K.Breuninger, and M. Pilkuhn, Phys. Stat. Sol.(a) 12, 169 (1972)

14) M. Lee, D.J. Nicholas, K.E.Singer, and B. Hamilton, J. Appl. Phys. 52, 2895 (1986)

15) M.C. Wu, and C.C. Chen, J.Appl. Phys. 72,4275 (1992)

16) D. Weiler and H. Mehrer, Philos. Mag. A 49,309 (1984)

17) J. Doerschel and U. Geissler, J. Crystal Growth. 121, 781 (1992) 